\title{
BMJ Open Effect of a smartphone application (Perx) on medication adherence and clinical outcomes: a 12-month randomised controlled trial
}

\author{
Ang Li D , , ${ }^{1,2}$ M Gail Del Olmo, ${ }^{1,3}$ Mackenzie Fong, ${ }^{1}$ Kyra Sim, ${ }^{1,3}$ Sharyn J Lymer, ${ }^{1}$ \\ Michelle Cunich, ${ }^{1,2}$ Ian Caterson ${ }^{1,3}$
}

To cite: Li A, Del Olmo MG, Fong $\mathrm{M}$, et al. Effect of a smartphone application (Perx) on medication adherence and clinical outcomes: a 12-month randomised controlled trial. BMJ Open 2021;11:e047041. doi:10.1136/ bmjopen-2020-047041

- Prepublication history for this paper is available online. To view these files, please visit the journal online (http://dx.doi. org/10.1136/bmjopen-2020047041).

Received 18 November 2020 Accepted 05 July 2021

Check for updates

(c) Author(s) (or their employer(s)) 2021. Re-use permitted under CC BY-NC. No commercial re-use. See rights and permissions. Published by BMJ.

${ }^{1}$ Boden Collaboration, Charles Perkins Centre, The University of Sydney, Sydney, NSW, Australia ${ }^{2}$ Sydney Health Economics Collaborative, Sydney Local Health District, Sydney, NSW, Australia

${ }^{3}$ Sydney Local Health District, Sydney, NSW, Australia

Correspondence to

Dr Ang Li;

ang.li5@unimelb.edu.au

\section{ABSTRACT}

Objective To determine whether the Perx app improves medication adherence and clinical outcomes over 12 months compared with standard care in patients requiring polypharmacy.

Design Randomised controlled trial with 12-month follow-up.

Setting Outpatient clinics in three tertiary hospitals in Sydney, Australia.

Participants Eligible participants were aged 18-75 years, with at least one chronic condition, taking $\geq 3$ different medications (oral medications or injections), with smartphone accessibility. Participants were randomised in a 1:1 ratio.

Interventions The intervention group used the Perx app that contained customised reminders and gamified interactions to reward verified medication adherence. Main outcome measures The primary outcome was medication adherence over 12 months measured using pill counts. Secondary outcomes included clinical outcomes (haemoglobin A1c ( $\mathrm{HbA1c})$, cholesterol, blood glucose, triglycerides, creatinine, thyroid function, blood pressure and weight).

Results 0 1412 participants screened for eligibility, 124 participants were randomised; 45 in the Perx arm and 40 in the control arm completed the study. The average age was $59.5,58.9 \%$ were women, chronic conditions were cardiovascular disease (78\%), type 2 diabetes $(75 \%)$, obesity (65\%) or other endocrine disorders (18\%). On average, participants were taking six medications daily. The Perx group had greater improvements in adherence at month 2 (Coef. 8\%; 95\% Cl 0.01 to 0.15), month 3 (Coef. $7 \%$; $95 \% \mathrm{Cl} 0.00$ to 0.14 ) and month 12 (Coef. $7 \% ; 95 \% \mathrm{Cl}$ 0.00 to 0.13 ). The probability of $\mathrm{HbA} 1 \mathrm{c} \leq 6.5 \%$ was greater in the Perx group at months 9 and 12 and cholesterol (total and low-density lipoprotein cholesterol) was lower in the Perx group at month 3 . The intervention was particularly effective for those with obesity, taking medications for diabetes and taking $\leq 4$ medications.

Conclusions This study provides evidence that app-based behavioural change interventions can increase medication adherence and produce longer-term improvements in some clinical outcomes in adults managing multimorbidity. More trials are needed to build the evidence base. Trial registration number ACTRN12617001285347.
Strengths and limitations of this study

- This study evaluates the long-term effectiveness of a medication adherence app in adults with multimorbidity, requiring polypharmacy, to manage chronic disease.

- Medication adherence was assessed with objective pill counts and validated with clinical outcomes rather than only self-reported questionnaires.

- Further research should focus on recruiting participants with poor control of their clinical measures to test the effectiveness of the app in the most severe multimorbid chronic disease cases.

- Future studies should consider including a 'selfcare' control group as a comparator where there is not any engagement with a health professional in monitoring medication adherence.

\section{INTRODUCTION}

Failure to take medication as prescribed is one of the recognised barriers to effective treatment of chronic illness. ${ }^{1}$ Medication non-adherence leads to poor control or worsening of disease with subsequent increased need for more medications or medical interventions, and greater risk of morbidity and mortality. ${ }^{2}$ Polypharmacy-concurrent use of multiple medications presents even a bigger challenge to medication adherence, due to increased regimen complexity. ${ }^{34}$ Over $30 \%$ of older Australians require polypharmacy, and therefore, identifying effective strategies to improve medication adherence is imperative. $^{5}$

Mobile health (mHealth) interventions are promising technologies to assist patients with adhering to their prescribed medication schedule. ${ }^{6-10}$ Smartphone applications (apps) offer a low cost and scalable health intervention modality; many people own a smartphone, and app development and delivery are relatively inexpensive. ${ }^{11}$ Over 5000 
medication adherence apps are available, but a relatively small body of evidence has evaluated their effectiveness. ${ }^{12}$ A recent meta-analysis found app users were more likely to adhere to prescribed medications than control participants but the median trial duration was only 12 weeks. ${ }^{13}$ Longer trials are needed to determine if adherence and clinical outcome improvements can be sustained.

Perx is a newly developed medication adherence app. ${ }^{14}$ Its features and functions include customisable medication regimen details and reminders, educational messages about disease management and healthcare visit prompts. The techniques employed throughout the app are based on behavioural science, gamification (trigger, action, reward and investment) and consumer engagement strategies to drive engagement and adherence, ${ }^{1516}$ and use several established behavioural science theories such as Prospect Theory, Hyperbolic Discounting, Mental Accounting and Anticipated Regret. ${ }^{17} 18$ Once adherence is verified through an in-app photo, users have an opportunity to earn rewards through gamified interactions. We investigated the association of medication adherence (the level of adherence rates and the indicator of adherence rates $\geq 90 \%$ ) and clinical outcomes (haemoglobin A1c (HbA1c), total cholesterol, high-density lipoprotein cholesterol (HDL-C), low-density lipoprotein cholesterol (LDL-C), triglycerides, fasting/non-fasting blood glucose (FBG/NFBG), creatinine, thyroid function tests (TFT), systolic and diastolic blood pressure and weight) with the Perx smartphone app compared with standard care in participants with chronic diseases requiring polypharmacy over 12 months. We hypothesised that the use of the reminder app would improve adherence and associated clinical outcomes.

\section{METHODS}

\section{Study design}

The Perx trial was a randomised controlled trial which investigated whether the Perx smartphone app use improves medication adherence and clinical outcomes in participants requiring polypharmacy over 12 months, compared with standard care. Enrolment began on 26 February 2018 and ended on 7 January 2019. The first participant commenced on 1 March 2018 and the last participant completed on 28 February 2020. All participants provided written informed consent. The trial was designed, and all procedures performed, independently of the study sponsor. Any prospective participants who are interested in using Perx can access the app at no cost for basic functions or a minimal cost for more advanced functions.

\section{Participants}

Individuals enrolled were aged $18-75$ years, had at least one chronic disease (type 2 diabetes, cardiovascular disease, heart failure, chronic kidney disease or other endocrine disorders), took a minimum of three different prescribed medications and had smartphone accessibility.

\begin{tabular}{|c|c|}
\hline Disease state & Common medications \\
\hline $\begin{array}{l}\text { Type } 2 \text { diabetes/ } \\
\text { endocrine } \\
\text { disorder }\end{array}$ & $\begin{array}{l}\text { Empagliflozin and metformin } \\
\text { hydrochloride, gliclazide, metformin, } \\
\text { metformin hydrochloride and sitagliptin } \\
\text { and metformin hydrochloride }\end{array}$ \\
\hline $\begin{array}{l}\text { Cardiovascular } \\
\text { disease/heart } \\
\text { failure }\end{array}$ & $\begin{array}{l}\text { Amlodipine, aspirin, atenolol, atorvastatin, } \\
\text { candesartan, clopidogrel, fenofibrate, } \\
\text { irbesartan, metoprolol, perindopril, } \\
\text { rosuvastatin, simvastatin and telmisartan }\end{array}$ \\
\hline $\begin{array}{l}\text { Chronic kidney } \\
\text { disease }\end{array}$ & $\begin{array}{l}\text { Cephalexin, dutasteride and tamsulosin, } \\
\text { finasteride, frusemide, methenamine } \\
\text { hippurate, mirabegron, oxybutynin } \\
\text { hydrochloride, solifenacin }\end{array}$ \\
\hline $\begin{array}{l}\text { Other endocrine } \\
\text { disease }\end{array}$ & $\begin{array}{l}\text { Fludrocortisone, hydrocortisone, } \\
\text { levothyroxine and liothyronine }\end{array}$ \\
\hline Others & $\begin{array}{l}\text { Amitriptyline, citalopram, desvenlafaxine, } \\
\text { esomeprazole, hydroxychloroquine, } \\
\text { methotrexate, mycophenolate, } \\
\text { pancrelipase, pantoprazole, pregabalin, } \\
\text { prednisone, rabeprazole, tacrolimus and } \\
\text { vitamin supplement }\end{array}$ \\
\hline
\end{tabular}

The prescribed medications are shown in table 1. Exclusion criteria were not owning a smartphone, unable to commit to the appointment schedule or not being able to speak or read English.

\section{Patient and public involvement}

Patients or the public were not involved in the design, or conduct, or reporting, or dissemination plans of our research.

\section{Recruitment and randomisation}

Participants were recruited through diabetes and heart failure clinics and the dialysis unit at Royal Prince Alfred Hospital, Concord Hospital and Liverpool Hospital (tertiary hospitals in Sydney, Australia) by the research nurse. Advertisements were placed on The University of Sydney and Perx websites and in the local newspapers. Registrants were screened and contacted if eligible. Potential participants from the Perx website were directed to a study website to assess eligibility and instructed to contact the researcher.

At the initial visit, conducted by the research nurse, eligible participants provided demographic data. Blood samples were collected after an overnight fast and pathology tests ordered depending on the participants' disease state/s (table 2). Blood pressure was measured using a Welch Allyn Connex Vital Signs digital sphygmomanometer. Weight was measured in light clothing to the nearest $0.1 \mathrm{~kg}$ using a calibrated digital scale. Obesity was diagnosed using the WHO criteria. ${ }^{19}$ Participants' prescribed medication regimen including disease state of the medication, indication, dosage, unit and frequency was also recorded. They were instructed to store their medications (including empty packets) in the medication 


\begin{tabular}{|c|c|c|}
\hline Disease state & Pathology test & Time points \\
\hline \multirow[t]{3}{*}{ Type 2 diabetes } & $\begin{array}{l}\text { Fasting blood } \\
\text { glucose }\end{array}$ & $\begin{array}{l}\text { Baseline and months } \\
3,6,9 \text { and } 12\end{array}$ \\
\hline & Haemoglobin A1c & \\
\hline & Lipid panel & \\
\hline $\begin{array}{l}\text { Cardiovascular } \\
\text { disease }\end{array}$ & Lipid panel & $\begin{array}{l}\text { Baseline and months } \\
3,6,9 \text { and } 12\end{array}$ \\
\hline $\begin{array}{l}\text { Other endocrine } \\
\text { disorder }\end{array}$ & $\begin{array}{l}\text { Thyroid function } \\
\text { test }\end{array}$ & $\begin{array}{l}\text { Baseline and months } \\
3,6,9 \text { and } 12\end{array}$ \\
\hline \multirow[t]{4}{*}{$\begin{array}{l}\text { Chronic kidney } \\
\text { disease }\end{array}$} & Serum creatinine & $\begin{array}{l}\text { Baseline and months } \\
3,6,9 \text { and } 12\end{array}$ \\
\hline & $\begin{array}{l}\text { Urine } \\
\text { microalbumin }\end{array}$ & $\begin{array}{l}\text { Baseline and month } \\
12\end{array}$ \\
\hline & Spot urine & $\begin{array}{l}\text { Baseline and month } \\
12\end{array}$ \\
\hline & $\begin{array}{l}\text { Albumin/creatinine } \\
\text { ratio }\end{array}$ & $\begin{array}{l}\text { Baseline and month } \\
12\end{array}$ \\
\hline
\end{tabular}

Notes: Lipid panel included tests for high-density lipoprotein cholesterol, low-density lipoprotein cholesterol and triglycerides. Other endocrine disorders included hypothyroidism, endocrine pancreatic insufficiency, adrenal insufficiency, thyroid cancer, hyperthyroidism and type 1 diabetes.

bag provided for the duration of the study and return it at clinic visits at months 1, 2, 3, 6, 9 and 12 of the study.

Participants were randomised to either the intervention (Perx) or standard care (control) group without stratification in a ratio of 1:1 using an online random number generator. Participants' usual healthcare providers were informed of their patients' participation in the trial but not group allocation. Due to the nature of the intervention, study participants and study staff (the research nurse) were not able to be blinded. The statisticians conducting the analyses were blinded to group allocation.

\section{Intervention}

Participants randomised to the Perx group downloaded the Perx app to their mobile device. They were educated and provided with written instructions on how to use the app. Participants set up a Perx profile and uploaded their medication schedule (medication name, medication appearance, dosage, unit and frequency).

On completing this, participants received customised reminders to take their tablets. They were prompted to take an in-app photo of their scheduled medication, in their hand, within 1 hour of the reminders to verify their expected medication schedule (medication name, medication appearance, dosage, unit and frequency). The uploaded photos for verification could not be saved onto the smart phone. A new photo must be uploaded for verification every time the participant took their medication. On verification of medication adherence, participants were eligible to win rewards through a gamified interaction. Redeemable gift cards were provided as incentives. Participants could interact with other Perx users through in-app forums and received weekly medication adherence reports. App costs were paid by the trial sponsor.

\section{Standard care}

Trial participants continued to attend routine visits with their healthcare providers as part of standard care. The control group did not access the Perx app during the trial. No other medication adherence apps were used, verified by the research nurse at each visit.

\section{Follow-Up assessment}

Follow-up assessments were performed for participants in both groups at months 1, 2, 3, 6, 9 and 12. Clinic visits at months 1, 2, 3, 6, 9 and 12 of the study were conducted at the research site by the research nurse who conducted pill counts (numbers of tablets taken and left). Clinic visits at months 3, 6, 9 and 12 included additional assessments of blood pressure, weight and collection of blood samples (table 2). All clinical and hospitalisation data were reviewed by the participants' doctor and the research team.

\section{Outcomes}

The primary outcome was overall medication adherence rate, defined as: number of dosage units taken/ (number of dosage units prescribed per day $\times$ number of prescribed days between two visits) for each individual, measured at months 1, 2, 3, 6, 9 and 12. Overall medication adherence was also assessed as a binary variable defined as: 1 : if overall medication adherence rates were $\geq 90 \%$ and 0 : otherwise. A valid cut-off for adherence should account for the specific drug, its pharmacological characteristics and disease severity. ${ }^{20}$ Considering the multimorbidity and polypharmacy nature of the participants, a frequently adopted and more strict threshold of $90 \%$ was chosen to avoid overestimation of the effect of the app on adherence. ${ }^{22}$ The secondary outcomes were changes in clinical outcomes (HbA1c, total cholesterol, HDL-C, LDL-C, triglycerides, FBG/NFBG, creatinine, TFT, systolic and diastolic blood pressure and weight) at baseline, months $3,6,9$ and 12 .

\section{Statistical analysis}

The trial enrolled 124 participants, 62 in each group. They were randomised to either the Perx group or control group in a ratio of $1: 1$ to have $90 \%$ power to detect a $20 \%$ (SD 40\%) difference in mean medication adherence between groups in an analysis of variance study of repeated measures with an alpha of 0.05 and allowing for a $20 \%$ loss to follow-up. ${ }^{22-24}$

Means and frequencies of baseline demographics were reported for any differences between the Perx and control groups. Medication adherence rate was estimated using pooled linear regression models with standard errors adjusted for within-individual correlation. Prespecified subgroup analyses for baseline body mass index (BMI) groups (a significant risk factor for chronic diseases ${ }^{25}$ disease state of the medication and number of medications were conducted as exploratory analyses. 
Table 3 Baseline demographics

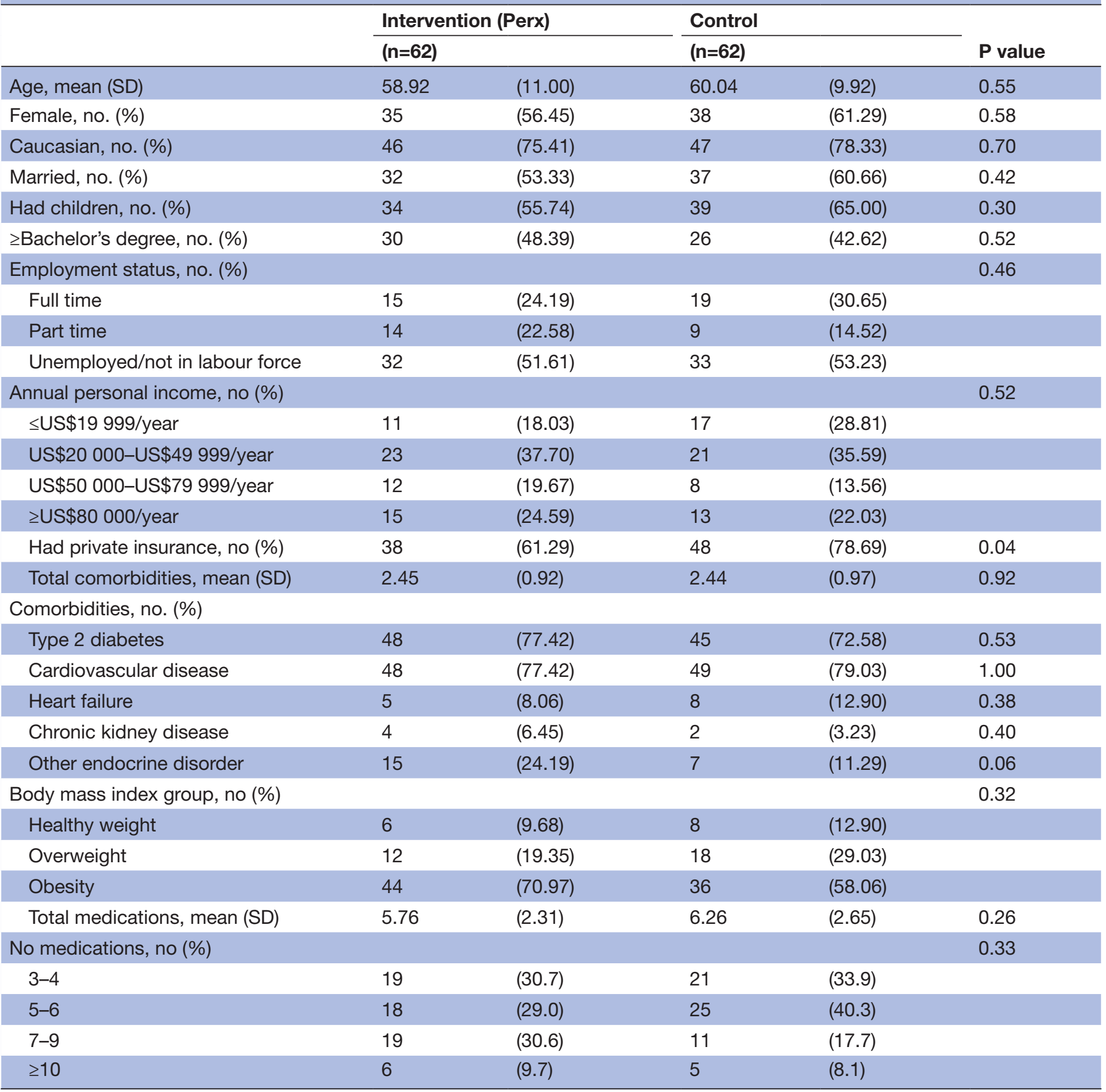

Binary medication adherence ( $\geq 90 \%$ or below) was estimated using pooled logistic regression models with adjusted SEs. The models included covariates for the visit number, treatment group and interactions between the visit number and treatment group. The primary analysis was conducted based on all available data.

Imputation approaches were also adopted as sensitivity analysis. Multiple imputation (10 imputations) using predictive mean matching method (three nearest neighbours) was performed to fill in missing values in the primary outcome variable. ${ }^{26}$ Baseline demographics (age, gender, ethnicity and marital status), socioeconomic (education level, employment status, annual income and private insurance status), health-related characteristics (number of medications, BMI group and specific comorbidity indicators), and medication adherence at other time points, were included as predictors.

Secondary analyses of continuous clinical outcomes (HbA1c, total cholesterol, HDL-C, LDL-C, triglycerides, FBG/NFBG, creatinine, TFT, blood pressure, weight and percentage weight change) were modelled using pooled linear regression models. Binary clinical outcomes (HbAlc $<7.0 \%$ and $\leq 6.5 \%$, total cholesterol $\leq 5.2 \mathrm{mmol} / \mathrm{L}$ and blood pressure $<135 / 85 \mathrm{~mm} \mathrm{Hg}$ ) were analysed using 


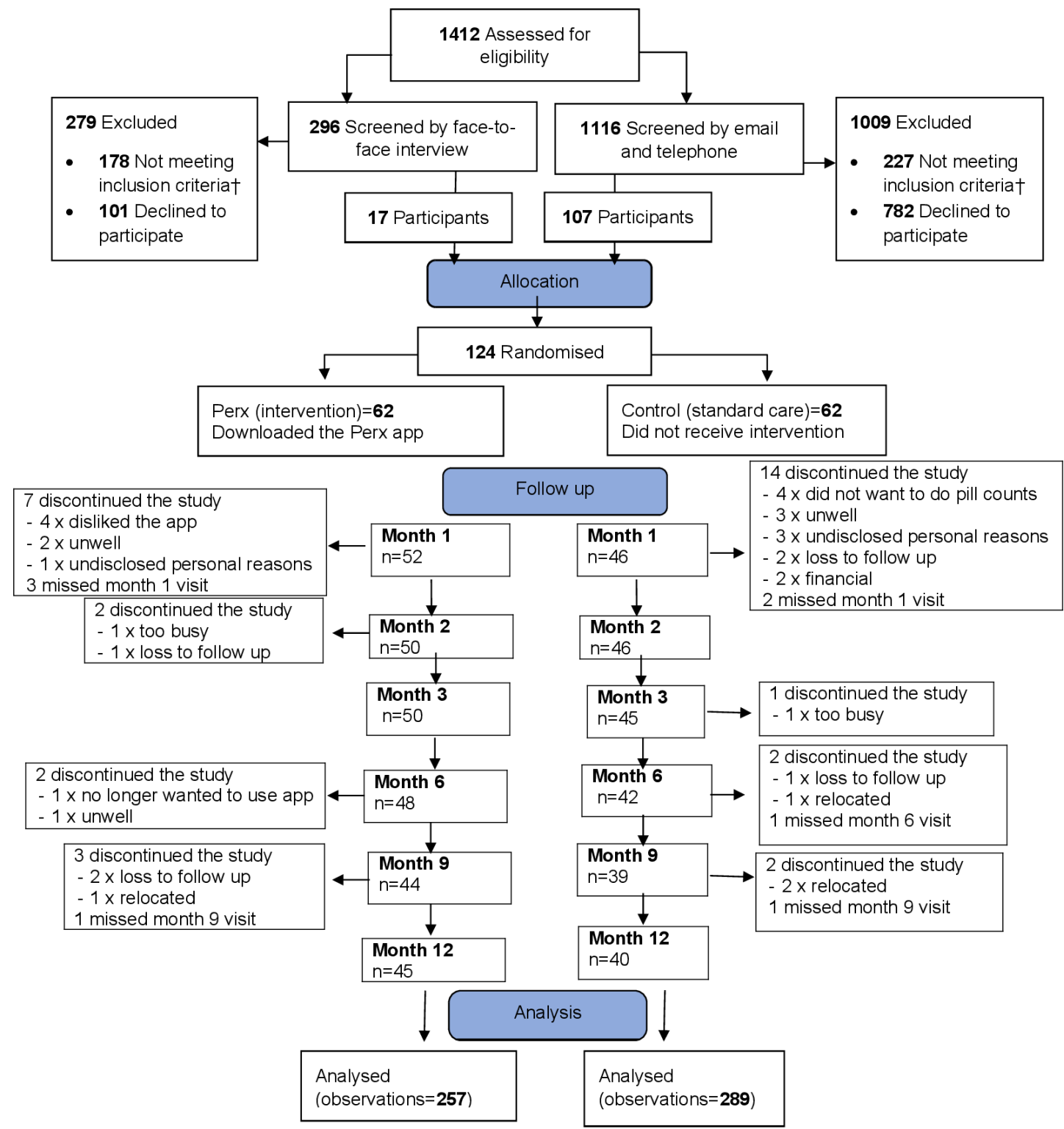

Notes: †The numbers not meeting inclusion criteria were not recorded as breakdown of different ineligibility reasons.

Figure 1 Consort study flow diagram.

pooled logistic regression models. Patients were analysed as allocated. The models for these clinical outcomes additionally controlled for baseline demographic factors (age, gender, ethnicity and marital status), socioeconomic factors (education level, employment status, annual income and private insurance status), clinical factors (BMI group and specific comorbidity indicators) and number of medications.

OR, 95\% CIs and $\mathrm{p}$ values are reported for binary outcomes and coefficient (Coef.), 95\% CIs and $p$ values are reported for continuous outcomes. Adjusted predictions of outcomes and $95 \%$ CIs are plotted in the figures (significant differences denoted with asterisks). Statistical significance was defined as $\mathrm{p}<0.05$. Power calculation was conducted in $\mathrm{G}^{*}$ power V.3.1 and analyses were conducted using Stata V.14.2 (StataCorp).

\section{RESULTS}

A total of 1412 potential participants were screened. Of the 124 eligible participants randomised at baseline, 52 in the Perx group and 45 in the control group attended the month 1 visit. This decreased to 45 in the Perx group and 40 in the control group at the 12-month visit. Baseline demographics are presented in table 3. Demographics and health-related characteristics of the Perx group were similar to the control group at the baseline and month 1 visits. Figure 1 shows the flow diagram of the study participants. While similar numbers of people in each group left the study due to ill health, relocation or undisclosed personal reasons (figure 1), it is possible that some people who left the Perx arm after the initial visit were less inclined to use mobile technology in general while people who stayed in the Perx arm or left the control 


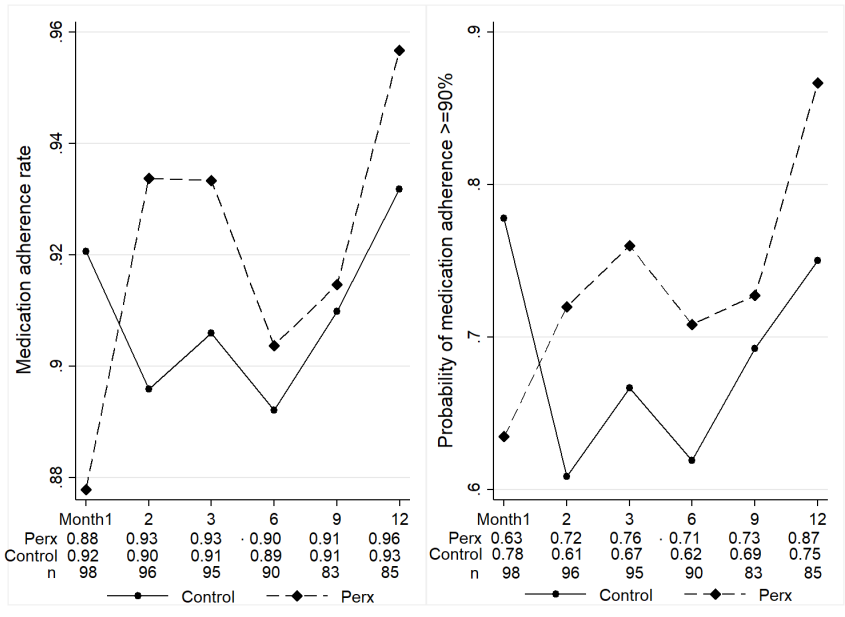

Figure 2 Overall medication adherence over time notes: average adherence rates in each arm, average probabilities of adherence $\geq 90 \%$ in each arm and numbers of observations at each time point are reported.

arm after the initial visit had predisposition towards using phones or apps. Changes in medication adherence rates and the probability of medication adherence $\geq 90 \%$ over time for the Perx and control groups are presented in figure 2, showing that adherence improved by the end of the study in the Perx group and remained relatively stable in the control group. No adverse events from using the app were reported. Participants had on average 89.5 sessions per month (3.0 per day) and 3.6 hours per month (7.3 min per day) in the Perx app.

\section{Patient baseline characteristics}

The average participant was aged 59.5 years; most were female $(73,58.9 \%)$.The participants had a mean BMI of $33.7 \mathrm{~kg} / \mathrm{m}^{2}$ and $80(64.5 \%)$ participants had obesity. Ninety-seven $(78.2 \%)$ had cardiovascular disease, 93 $(75.0 \%)$ had type 2 diabetes, 22 (17.7\%) had other endocrine disorders (hypothyroidism (15), endocrine pancreatic insufficiency (2), adrenal insufficiency (2), thyroid cancer (1), hyperthyroidism (1) or type 1 diabetes (1)),13 (10.5\%) had heart failure and $6(4.8 \%)$ had chronic kidney disease. Thirty-seven participants $(29.8 \%)$ had one condition, $71(57.3 \%)$ had two conditions, 13 $(10.5 \%)$ had three conditions and $3(2.4 \%)$ had four or five conditions. On average, participants took six medications per day (range 3-15); 32.3\% took 3-4 medications, $34.7 \% 5-6,24.2 \%$ took $7-9$ and $8.9 \%$ took 10 or more.

\section{Effects of interventions on total medication adherence}

The probability of adhering to medication were $8 \%$ higher in the Perx group than in the control group at month 2 (95\% CI 0.01 to $0.15 ; \mathrm{p}=0.025)$, $7 \%$ higher at month 3 (95\% CI 0.00 to $0.14 ; \mathrm{p}=0.046)$ and $7 \%$ higher at month 12 (95\% CI 0.00 to 0.13 ; $\mathrm{p}=0.044$ ), compared with month 1 (table 4). Multiple imputation analyses that accounted for missing data (34 observations imputed) produced consistent results at months 2 and 12 . The significant increase in the odds of adherence $\geq 90 \%$ were 3.33 times higher (95\% CI 1.02 to $10.84 ; \mathrm{p}=0.046)$ at month 2 and 4.37 times higher (95\% CI 1.07 to $17.78 ; \mathrm{p}=0.040$ ) at month 12 for the Perx group than for the control group. Multiple imputation analyses produced consistent results at month 12. No significant improvement was observed at months 6 and 9. Results additionally controlling for baseline demographic (age, gender, ethnicity and marital status), socioeconomic (education level, employment status, annual income and private insurance status), clinical (BMI group and specific comorbidity indicators) and medication count covariates were similar.

\section{Effects of interventions on medication adherence by subgroups}

Exploratory subgroup analyses by BMI category, disease state of the medication, and number of medications are presented in table 5 . The Perx app significantly improved medication adherence in participants with obesity at month 2 (Coef. 13\%; 95\% CI 0.04 to 0.22 ; $\mathrm{p}=0.004$ ), month 3 (Coef. 12\%; 95\% CI 0.04 to $0.20 ; \mathrm{p}=0.004$ ) and month 6 (Coef. 12\%; 95\% CI 0.02 to $0.22 ; \mathrm{p}=0.018$ ).

The intervention was most effective for participants taking a smaller number of medications (3-4) at month 2 (Coef. 0.19; 95\% CI 0.06 to $0.31 ; \mathrm{p}=0.004$ ), month 3 (Coef. 0.15 ; $95 \%$ CI 0.04 to $0.26 ; \mathrm{p}=0.009$ ), month 6 (Coef. 0.15 ; $95 \%$ CI 0.04 to $0.26 ; \mathrm{p}=0.006$ ) and month 12 (Coef. 0.13; $95 \%$ CI 0.03 to $0.23 ; \mathrm{p}=0.008$ ).

The increase in adherence to medications for diabetes or kidney disease was significantly higher in the Perx group than controls at months 6 (Coef. 0.13; 95\% CI 0.02 to $0.24 ; \mathrm{p}=0.022$ ) and 12 (Coef. $0.11 ; 95 \%$ CI 0.01 to 0.20 ; $\mathrm{p}=0.028$ ).

\section{Effects of interventions on clinical outcomes}

Figure 3 shows the changes in clinical outcomes at each visit over time for the treatment and control groups. The probability of $\mathrm{HbA} 1 \mathrm{c} \leq 6.5 \%$ was significantly higher in the Perx group than the control group at month 9 (difference in probability $0.30 ; 95 \%$ CI 0.03 to $0.48 ; \mathrm{p}=0.027$ ) and month 12 (difference in probability $0.22 ; 95 \% \mathrm{CI}$ 0.01 to $0.44 ; \mathrm{p}=0.039$ ) (panel (a)). The probability of total cholesterol $\leq 5.2 \mathrm{mmol} / \mathrm{L}$ was significantly higher in the Perx group at month 3 (difference in probability $0.16 ; 95 \%$ CI, -0.01 to $-0.30 ; \mathrm{p}=0.031$ ) (panel (b)). The level of LDL-C was significantly lower in the Perx group at month 3 (difference in level -0.58 ; $95 \%$ CI -1.07 to -0.09 ; $\mathrm{p}=0.019)($ panel $(\mathrm{c}))$.

There were no significant differences in FBG, NFBG, creatinine, TFT (panel (d)), blood pressure (panel (e)) or weight or percentage weight change (panel (f)) between the Perx and control groups over the study period.

\section{DISCUSSION}

\section{Principal findings}

This study evaluates the long-term effectiveness of a medication adherence app in adults requiring polypharmacy to manage multiple chronic diseases. The intervention 
Table 4 Effects of the Perx app on medication adherence over time

\begin{tabular}{|c|c|c|c|c|c|c|c|c|}
\hline & \multicolumn{8}{|c|}{ Medication adherence rates } \\
\hline & \multicolumn{4}{|c|}{ All available data } & \multicolumn{4}{|c|}{ Multiple imputation } \\
\hline & Coef. & $P$ value & \multicolumn{2}{|l|}{$95 \% \mathrm{Cl}$} & Coef. & $P$ value & \multicolumn{2}{|l|}{$95 \% \mathrm{Cl}$} \\
\hline \multicolumn{9}{|l|}{ Base: M1 } \\
\hline M2 & -0.02 & 0.320 & -0.07 & 0.02 & -0.02 & 0.330 & -0.08 & 0.03 \\
\hline M3 & -0.01 & 0.532 & -0.06 & 0.03 & -0.02 & 0.511 & -0.06 & 0.03 \\
\hline M9 & -0.01 & 0.691 & -0.06 & 0.04 & -0.01 & 0.719 & -0.06 & 0.04 \\
\hline M12 & 0.01 & 0.580 & -0.03 & 0.05 & 0.01 & 0.705 & -0.03 & 0.05 \\
\hline Base: Perx & -0.04 & 0.154 & -0.10 & 0.02 & -0.04 & 0.154 & -0.10 & 0.02 \\
\hline M2 x Perx & 0.08 & 0.025 & 0.01 & 0.15 & 0.08 & 0.028 & 0.01 & 0.15 \\
\hline M3 x Perx & 0.07 & 0.046 & 0.00 & 0.14 & 0.07 & 0.066 & 0.00 & 0.14 \\
\hline \multirow[t]{4}{*}{ M12 x Perx } & 0.07 & 0.044 & 0.00 & 0.13 & 0.07 & 0.049 & 0.00 & 0.14 \\
\hline & \multicolumn{8}{|c|}{ Medication adherence rates $\geq 90 \%$} \\
\hline & \multicolumn{4}{|c|}{ All available data } & \multicolumn{4}{|c|}{ Multiple imputation } \\
\hline & OR & $P$ value & $95 \% \mathrm{CI}$ & & OR & $P$ value & $95 \% \mathrm{Cl}$ & \\
\hline \multicolumn{9}{|l|}{ Base: M1 } \\
\hline M2 & 0.44 & 0.044 & 0.20 & 0.98 & 0.47 & 0.064 & 0.21 & 1.05 \\
\hline M3 & 0.57 & 0.183 & 0.25 & 1.30 & 0.60 & 0.226 & 0.26 & 1.38 \\
\hline M6 & 0.46 & 0.134 & 0.17 & 1.26 & 0.47 & 0.137 & 0.17 & 1.27 \\
\hline M9 & 0.64 & 0.258 & 0.30 & 1.38 & 0.62 & 0.238 & 0.28 & 1.37 \\
\hline M12 x Perx & 4.37 & 0.040 & 1.07 & 17.78 & 4.95 & 0.041 & 1.07 & 22.84 \\
\hline No observations & 546 & & & & 580 & & & \\
\hline
\end{tabular}

Notes: Generalised linear models were estimated. Bold estimates indicate significance at the $5 \%$ level. app, application; Coef., coefficient; m, month.;

was designed to be scalable and sustainable for implementation. Improvements in medication adherence were observed at months 2, 3 and 12. Improvements in total cholesterol and LDL-C appeared at month 3 and improvements in blood glucose control persisted until the end of the trial at months 9 and 12. The findings indicate the potential efficacy of mobile device apps in improving medication adherence and some clinical outcomes among adults managing multimorbidity.

The effectiveness of the intervention was also related to BMI status, disease state of the medication and number of medications. Significant and large improvements in medication adherence were shown for participants with obesity at months 2, 3 and 6. Consistent with the app's longer-term efficacy on blood glucose control, adherence to medications for diabetes and kidney disease showed significant improvements at months 6 and 12. The app was most effective for participants who took a relatively smaller number of medications at months 2, 3, 6 and 12.

\section{Comparison with previous studies}

Recent meta-analyses on the efficacy of mobile device apps indicated greater medication adherence among app users. ${ }^{1327}$ However, the majority of studies were based on self-reported measures of adherence and targeted single health condition, and research with longer duration was lacking. ${ }^{1327}$ This study provides novel evidence for the longer-term efficacy of medication adherence apps on medication adherence and some clinical outcomes. Similar randomised controlled trials have reported no 
Table 5 Effects of the Perx app on medication adherence by subgroups

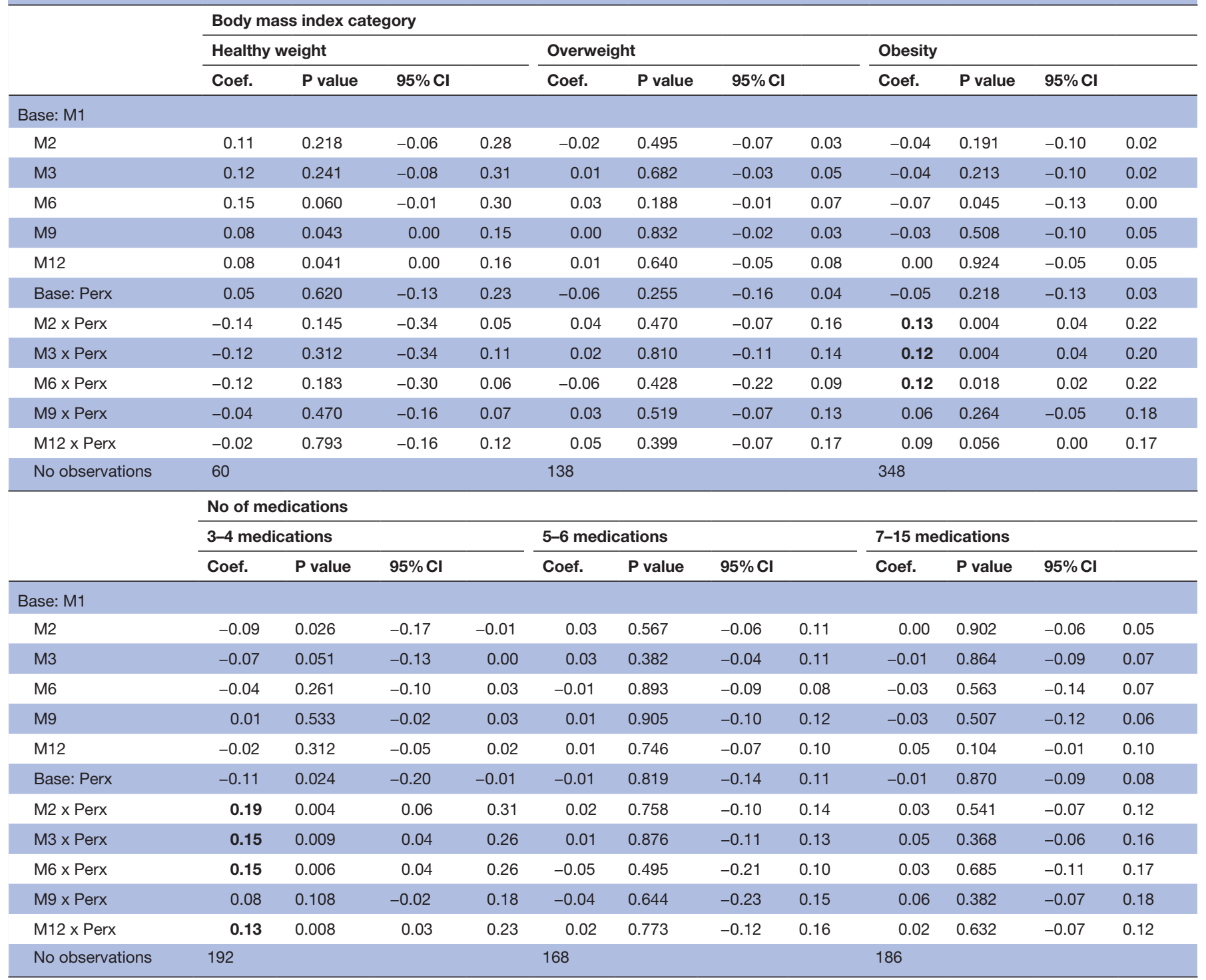

\begin{tabular}{|c|c|c|c|c|c|c|c|c|c|c|c|c|}
\hline \multirow{3}{*}{ Base: M1 } & \multicolumn{12}{|c|}{ Disease state of medications } \\
\hline & \multicolumn{4}{|c|}{ Cardiovascular disease } & \multicolumn{4}{|c|}{ Type 2 diabetes/kidney disease } & \multicolumn{4}{|c|}{ Other endocrine disorder/others } \\
\hline & Coef. & $P$ value & \multicolumn{2}{|l|}{$95 \% \mathrm{Cl}$} & Coef. & $P$ value & \multicolumn{2}{|l|}{$95 \% \mathrm{Cl}$} & Coef. & $P$ value & \multicolumn{2}{|l|}{$95 \% \mathrm{Cl}$} \\
\hline M2 & 0.00 & 0.858 & -0.04 & 0.05 & -0.04 & 0.066 & -0.09 & 0.00 & -0.01 & 0.744 & -0.08 & 0.06 \\
\hline M3 & 0.01 & 0.647 & -0.04 & 0.06 & 0.00 & 0.917 & -0.06 & 0.06 & 0.00 & 0.950 & -0.07 & 0.07 \\
\hline M6 & -0.01 & 0.730 & -0.06 & 0.05 & -0.08 & 0.050 & -0.15 & 0.00 & 0.02 & 0.564 & -0.05 & 0.09 \\
\hline M12 & 0.03 & 0.132 & -0.01 & 0.06 & -0.02 & 0.591 & -0.09 & 0.05 & 0.04 & 0.237 & -0.03 & 0.11 \\
\hline Base: Perx & 0.01 & 0.821 & -0.05 & 0.07 & -0.05 & 0.241 & -0.12 & 0.03 & 0.02 & 0.640 & -0.06 & 0.10 \\
\hline M2 x Perx & 0.01 & 0.684 & -0.05 & 0.07 & 0.08 & 0.053 & 0.00 & 0.16 & 0.03 & 0.539 & -0.06 & 0.12 \\
\hline M3 x Perx & 0.01 & 0.824 & -0.06 & 0.08 & 0.03 & 0.520 & -0.06 & 0.11 & 0.04 & 0.377 & -0.05 & 0.13 \\
\hline M6 x Perx & 0.02 & 0.575 & -0.06 & 0.10 & 0.13 & 0.022 & 0.02 & 0.24 & 0.01 & 0.896 & -0.09 & 0.10 \\
\hline
\end{tabular}

Notes: Generalised linear models were estimated. Bold estimates indicate significance at the $5 \%$ level.

app, application; Coef., coefficient.; 

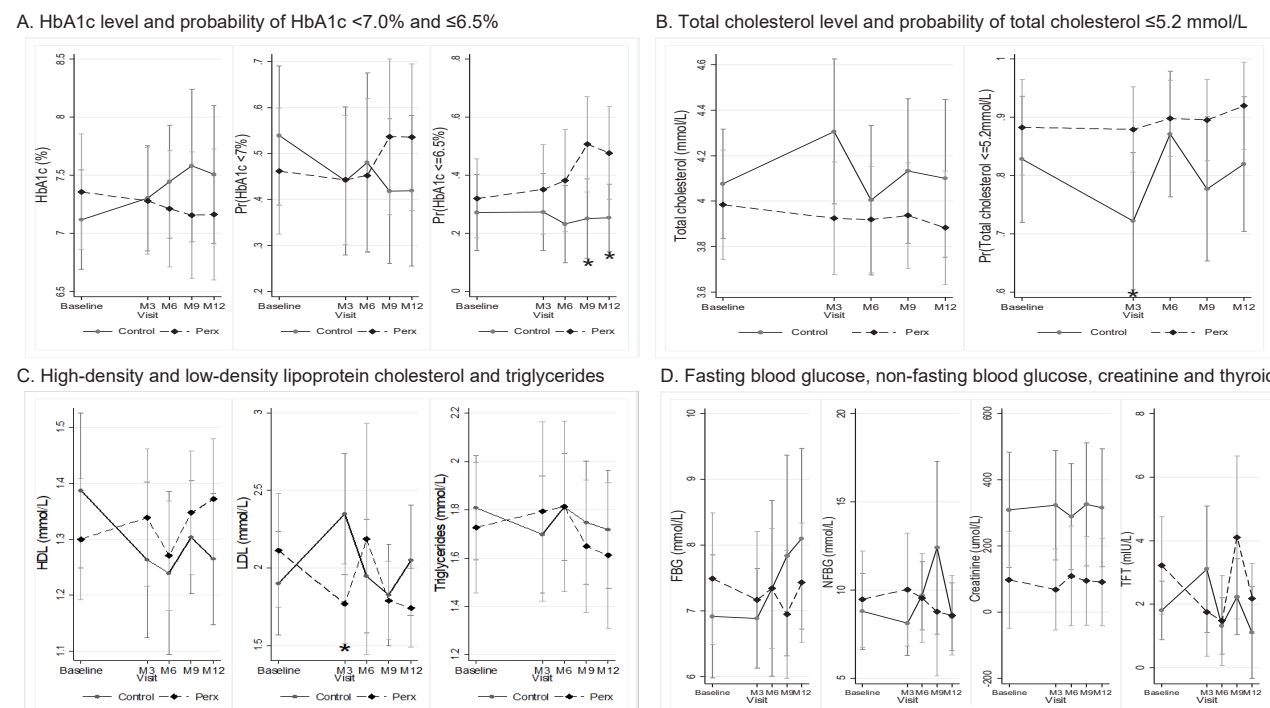

D. Fasting blood glucose, non-fasting blood glucose, creatinine and thyroid function test
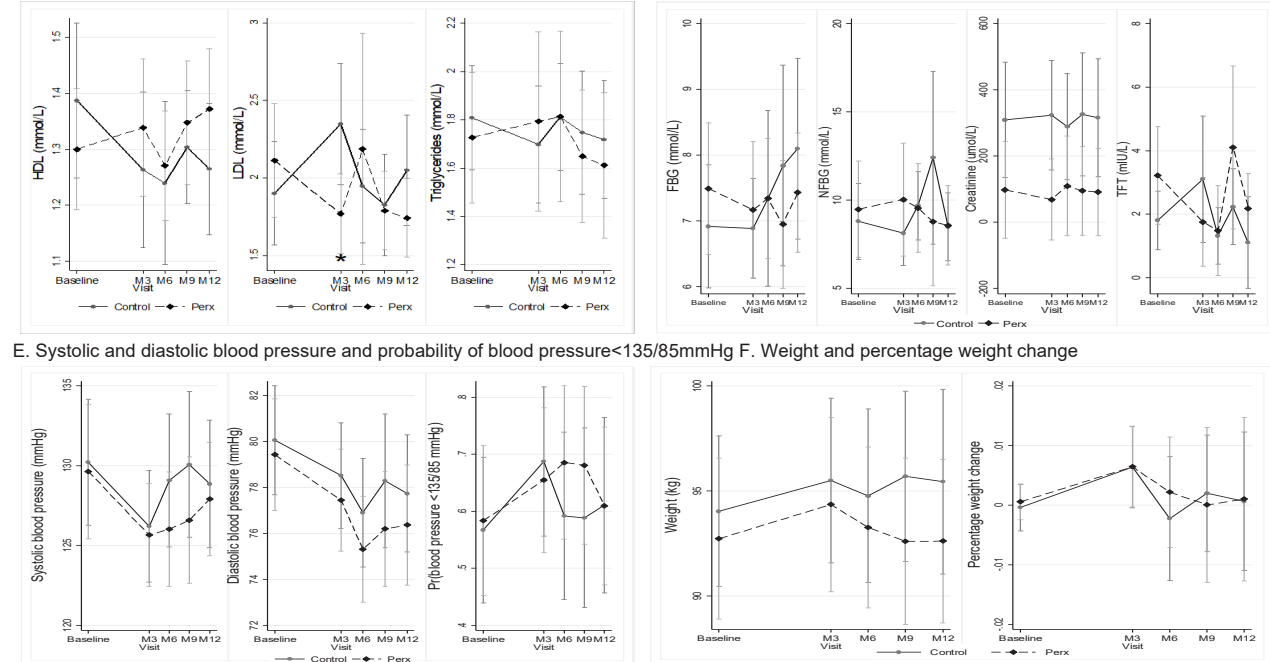

Figure 3 Effects of the Perx app on clinical outcomes notes: generalised linear models were estimated, with SEs adjusted, controlling for baseline demographic, socioeconomic and clinical characteristics and medication counts. Linear links were used for continuous outcomes and logit links were used for binary outcomes. Coefficients or average marginal effects were estimated and adjusted predictions of outcomes and $95 \%$ Clls were plotted. Significant differences between groups at the $5 \%$ level are denoted with asterisks. The model for creatinine might lack precision as only 59 observations were available. FBG, fasting blood glucose; HbA1c, haemoglobin A1c; HDL, high-density lipoprotein cholesterol; LDL; low-density lipoprotein cholesterol; M, month; NFBG, non-fasting blood glucose; TFT, thyroid function test.

difference in clinical outcomes between intervention and control groups during the initial months of app use. ${ }^{128-31}$ However, the current study shows that group differences in total cholesterol and LDL-C were significant at month 3 , and improvements in HbAlc in Perx users became apparent from month 9 onwards.

\section{Explanations and implications}

The long-term improvements in some clinical outcomes such as blood glucose control suggest the potential of mobile device apps as effective interventions to promote medication adherence. It is pertinent that the differences in some clinical outcomes became apparent when study visits were less frequent. While the adherence was lower in the Perx group at month 1, the app significantly improved the adherence in the Perx group compared with the control group over the short term at months 2 and 3 and long term at month 12, although both groups had improvement at month 12 that may be due to increased salience of the trial in the final visit. Apps may be a convenient and effective tool to enhance and sustain medication adherence especially between healthcare visits that may be up to several months apart.
Large improvements in medication adherence for participants with obesity indicate the usefulness of the app for individuals who are more likely to have multimorbidity, ${ }^{32}$ and require more healthcare and complex treatment plans. Significant improvements were also observed among participants with type 2 diabetes or kidney disease. This could be because the medications associated with these disease conditions tend to produce more noticeable effects that motivate adherence, or because regular blood tests are generally required for these participants who can observe the benefit of adherence more tangibly and hence are more likely to adhere with the assistance of the app.

The lower effectiveness of the app for treatment regimens with more medications may be explained by forgetfulness, confusion, and cognitive or pill burden associated with frequent and complex medication regimens, ${ }^{33}$ and the extra time required interacting with the app and the 'nuisance' of photo taking (quote by participants) and pill counting. The amount of time required to engage with the app is an important factor associated with effective health behavioural change. ${ }^{27}$ Modifying the app so that the verification process is less burdensome 
could help to extend the benefits to participants taking many tablets.

A smartphone app such as Perx can be implemented in a non-research clinical setting for routine use in practice. Interested mobile phone users can download and use the app, although embedding more languages and screen reader features will increase the accessibility of the app. Measures should be in place to ensure that the app does not contribute to health inequalities for people who do not have access or have language barriers. Additionally, linking pharmacy and general practice services with the app can potentially increase the effectiveness of the app. More qualitative and implementation studies such as a cost-effectiveness analysis from a healthcare provider perspective may need to be considered to assess and ensure the feasibility and efficacy of the app on a larger scale.

\section{Strengths and limitations}

The efficacy of a mobile phone app was assessed for adherence to polypharmacy using a randomised controlled trial over long intervention duration. Medication adherence was assessed objectively, and numerous clinical outcomes were measured over the study period. The study focused on a population with multimorbidity requiring polypharmacy who were at higher risks of incurring avoidable hospitalisations and preventable complications. ${ }^{32}{ }^{34}$ Subgroup analyses and multiple imputation were conducted on the primary study outcome to explore effect heterogeneity and assess result robustness.

The study had some limitations. First, baseline measures of adherence were not assessed. The model could, therefore, not include a preintervention measure; however, demographics at baseline and month 1 were evenly distributed following randomisation. Second, returning medication bags to the research nurse at study visits may have promoted self-monitoring in both groups, potentially impacting on the study's findings and generalisability. However, the frequency of later visits was similar to that in real life primary healthcare settings. Including a 'self-care' control group that does not engage in any selfmonitoring activities would be of value to future studies. Third, baseline glycaemic control among all participants was generally good, potentially reducing the generalisability of findings. Good glycaemic control could be attributed to participants' relatively high education status. Fourth, at the time of the study, the app was exclusively online, limiting accessibility when participants were not connected to the internet. Offline functionality has since been developed and could plausibly increase the effect size of the intervention. Fifth, this study evaluated a single app therefore its findings may not be generalisable to all medication adherence apps. Sixth, it was possible for some participants to reveal their group allocation to their healthcare providers. Seventh, people who stayed in the Perx arm or left the control arm after the initial visit potentially had predisposition towards using phones or apps. Lastly, while pill count is a more objective measure of adherence than self-reported questionnaires, participants could plausibly discard tablets to feign adherence. Measuring clinical outcomes in the current study helped to validate adherence.

\section{CONCLUSION}

This study provides preliminary evidence that app-based behavioural change interventions can increase medication adherence and produce longer-term improvements in some clinical outcomes in adults managing chronic disease with polypharmacy. These interventions may be particularly effective for those with obesity, taking medications for type 2 diabetes and for people taking a relatively smaller number of medications. More trials are needed to build the evidence base.

\section{Twitter Ang Li @ANGLIhere}

Contributors IC had full access to all of the data in the study and takes responsibility for the integrity of the data and the accuracy of the data analysis. Study concept and design: MF, KS, SJL, MC and IC. Acquisition, analysis or interpretation of data: $A L, M G D O$, SJL and MC. Drafting of the manuscript: AL, MGDO, MF, KS, SJL and MC. Critical revision of the manuscript for important intellectual content: AL, MGDO, MF, KS, SJL, MC and IC. Statistical analysis: AL, MF, SJL, MC. Administrative, technical or material support: MGDO and KS. Study supervision: IC.

Funding This work was supported by funding from the Sydney Local Health District (BR17/1646). The app was provided by Perx Health.

Disclaimer Sydney Local Health District had no role in the conduct of the study; collection, management, analysis, or interpretation of the data; preparation, review, or approval of the manuscript; or decision to submit the manuscript for publication.

Competing interests None declared.

Patient and public involvement Patients and/or the public were not involved in the design, or conduct, or reporting, or dissemination plans of this research.

Patient consent for publication Not required.

Ethics approval The study was approved by the Ethics Review Committee (RPAH Zone) of the Sydney Local Health District (X17-0229).

Provenance and peer review Not commissioned; externally peer reviewed.

Data availability statement Data are available on reasonable request. The data that support the findings of this study are available on reasonable request from the corresponding author.

Open access This is an open access article distributed in accordance with the Creative Commons Attribution Non Commercial (CC BY-NC 4.0) license, which permits others to distribute, remix, adapt, build upon this work non-commercially, and license their derivative works on different terms, provided the original work is properly cited, appropriate credit is given, any changes made indicated, and the use is non-commercial. See: http://creativecommons.org/licenses/by-nc/4.0/.

ORCID iD

Ang Li http://orcid.org/0000-0002-6269-6432

\section{REFERENCES}

1 Nasseh K, Frazee SG, Visaria J. Cost of medication nonadherence associated with diabetes, hypertension, and dyslipidemia. Am J Pharm Benefits 2012;4:e41-7.

2 Ho PM, Magid DJ, Shetterly SM, et al. Medication nonadherence is associated with a broad range of adverse outcomes in patients with coronary artery disease. Am Heart J 2008;155:772-9.

3 Cantlay A, Glyn T, Barton N. Polypharmacy in the elderly. InnovAiT 2016;9:69-77.

4 Marcum ZA, Gellad WF. Medication adherence to multidrug regimens. Clin Geriatr Med 2012;28:287-300. 
5 Page AT, Falster MO, Litchfield M, et al. Polypharmacy among older Australians, 2006-2017: a population-based study. Medical Journal of Australia 2019;211:71-5.

6 Choudhry NK, Krumme AA, Ercole PM, et al. Effect of reminder devices on medication adherence: the remind randomized clinical trial. JAMA Intern Med 2017;177:624-31.

7 Bailey SC, Belter LT, Pandit AU, et al. The availability, functionality, and quality of mobile applications supporting medication selfmanagement. J Am Med Inform Assoc 2014;21:542-6.

8 De Jongh T, Gurol-Urganci I, Vodopivec-Jamsek V. Mobile phone messaging for facilitating self-management of long-term illnesses. Cochrane Database of Systematic Reviews 2012;12.

9 Patrick K, Griswold WG, Raab F, et al. Health and the mobile phone. Am J Prev Med 2008;35:177-81.

10 Li A, Cunich M, Fuller N, et al. Improving adherence to weight-loss medication (liraglutide $3.0 \mathrm{Mg}$ ) using mobile phone text messaging and healthcare professional support. Obesity 2020;28:1889-901.

11 Ahmed I, Ahmad NS, Ali S, et al. Medication adherence apps: review and content analysis. JMIR Mhealth Uhealth 2018;6:e62.

12 Morawski K, Ghazinouri R, Krumme A, et al. Association of a smartphone application with medication adherence and blood pressure control: the MedISAFE-BP randomized clinical trial. JAMA Intern Med 2018;178:802-9.

13 Armitage LC, Kassavou A, Sutton S. Do mobile device apps designed to support medication adherence demonstrate efficacy? A systematic review of randomised controlled trials, with metaanalysis. BMJ Open 2020;10:e032045.

14 Perx Health. Perx health: better health engagement and outcomes, 2018. Available: https://perxhealth.com/

15 Loewenstein G, Asch DA, Friedman JY, et al. Can behavioural economics make us healthier? BMJ 2012;344:e3482.

16 Eyal N. Hooked: how to build habit-forming products. New York: Penguin, 2014.

17 Dhami S. The foundations of behavioral economic analysis. Oxford: Oxford University Press, 2016.

18 Wilkinson N, Klaes M. An introduction to behavioral economics. London, UK: Macmillan International Higher Education, 2017.

19 Organization WH. Obesity: preventing and managing the global epidemic. Geneva: World Health Organization, 2000.

20 Hartman L, Lems WF, Boers M. Outcome measures for adherence data from a medication event monitoring system: a literature review. J Clin Pharm Ther 2019;44:1-5.
21 Burnier $M$. Is there a threshold for medication adherence? lessons learnt from electronic monitoring of drug adherence. Front Pharmacol 2018;9:1540.

22 Thakkar J, Kurup R, Laba T-L, et al. Mobile telephone text messaging for medication adherence in chronic disease: a meta-analysis. JAMA Intern Med 2016;176:340-9.

23 Kini V, Ho PM. Interventions to improve medication adherence: a review. JAMA 2018;320:2461-73.

24 Tao D, Xie L, Wang T, et al. A meta-analysis of the use of electronic reminders for patient adherence to medication in chronic disease care. J Telemed Telecare 2015;21:3-13.

25 Australian Institute of Health and Welfare. Impact of overweight and obesity as a risk factor for chronic conditions. Canberra: Australian Institute of Health and Welfare, Australian Government, 2017.

26 Rubin DB. Statistical matching using file concatenation with adjusted weights and multiple imputations. Journal of Business \& Economic Statistics 1986;4:87-94

27 Zhao J, Freeman B, Li M. Can mobile phone Apps influence people's health behavior change? an evidence review. J Med Internet Res 2016;18:e287.

28 Park LG, Howie-Esquivel J, Chung ML, et al. A text messaging intervention to promote medication adherence for patients with coronary heart disease: a randomized controlled trial. Patient Educ Couns 2014;94:261-8.

29 DeKoekkoek T, Given B, Given CW, et al. mHealth SMS text messaging interventions and to promote medication adherence: an integrative review. J Clin Nurs 2015;24:2722-35.

30 Santo K, Singleton A, Rogers K, et al. Medication reminder applications to improve adherence in coronary heart disease: a randomised clinical trial. Heart 2019;105:323-9.

31 Peng $\mathrm{Y}$, Wang $\mathrm{H}$, Fang $\mathrm{Q}$, et al. Effectiveness of mobile applications on medication adherence in adults with chronic diseases: a systematic review and meta-analysis. J Manag Care Spec Pharm 2020;26:550-61.

32 Druss BG, Marcus SC, Olfson M, et al. Comparing the National economic burden of five chronic conditions. Health Aff 2001;20:233-41.

33 Brown M, Sinsky CA. Medication adherence: we didn't ask and they didn't tell. Fam Pract Manag 2013;20:25-30.

34 Wolff JL, Starfield B, Anderson G, Prevalence AG. Prevalence, expenditures, and complications of multiple chronic conditions in the elderly. Arch Intern Med 2002;162:2269-76. 